\section{Dr. Masiero replies}

\section{To the Editor:}

The aim of the letter by Colina, et $a l^{1}$ is to determine the role of rehabilitation in combination with tumor necrosis factor (TNF) inhibitor therapy in the treatment of ankylosing spondylitis (AS). The results of the study by Colina, et $a l^{2}$ on quality of life, as measured by the EQ-5D visual analog scale, are further evidence in support of the usefulness of combining TNF inhibitor therapy and intensive spa rehabilitation in patients with AS. In our recent work ${ }^{3}$, we demonstrated that combining exercise with an educational-behavioral program can give promising results in the management of patients with clinically stabilized AS receiving approved TNF inhibitor treatment. We suppose that the positive results of rehabilitation intervention depend on many factors correlated to the positive effects of TNF inhibitors, which can provide symptom relief, delaying spinal inflammation, and improve compliance. Dubey, et $a l^{4}$ showed that motivation levels and time spent on physical therapy can improve significantly in patients undergoing TNF inhibitor treatment. In our study we introduced an educational-behavioral program in association with a simple exercise training protocol designed for patients at home. We agree that rehabilitation combined with an educational-behavioral program could represent an effective choice for AS patients in remission. To date, however, optimal management of patients with AS has not been determined and prudence is still required in defining the potential role of rehabilitation intervention in combination with TNF inhibitor therapy. Although TNF inhibitor therapy has dramatically changed the therapeutic opportunities for patients with AS by providing symptom relief and consequently improving quality of life, it is a source of great expense for the national health service. In this perspective, the search for a new approach combining rehabilitation with an educational-behavioral program could represent an effective choice for AS patients in remission after discontinuation of TNF inhibitor therapy.

We believe that the rehabilitation "challenge" for the future in AS treatment with TNF inhibitor therapy should be: (1) to define optimal rehabili- tation protocols to be recommended in the management of AS patients in the active, remission, or stabilized phase; (2) to introduce adequate assessment measures to more specifically quantify rehabilitation outcome in association with measures currently used in the rheumatology setting; (3) to assess whether rehabilitation approaches can reduce the consumption of TNF inhibitor therapy while maintaining the same beneficial effects on functionality and quality of life; and (4) to evaluate whether, with the same pharmacological treatment with TNF inhibitors, rehabilitation intervention has a positive influence on the radiological progression of AS.

STEFANO MASIERO, MD, Rehabilitation Unit, Department of Rehabilitation Medicine, University of Padua, Via Giustiniani 2, 35128 Padua, Italy. Address correspondence to Dr. Masiero;

E-mail: stef.masiero@unipd.it

\section{REFERENCES}

1. Colina M, Ciancio G, Govoni M. Rehabilitation treatment strategies in ankylosing spondylitis [letter]. J Rheumatol 2011;38:2266.

2. Colina M, Ciancio G, Garavini R, Conti M, Trotta F, Govoni M. Combination treatment with etanercept and an intensive spa rehabilitation program in active ankylosing spondylitis. Int J Immunopathol Pharmacol 2009;22:1125-9.

3. Masiero S, Bonaldo L, Pigatto M, Lo Nigro A, Ramonda R, Punzi L. Rehabilitation treatment in patients with ankylosing spondylitis stabilized with tumor necrosis factor inhibitor therapy. A randomized controlled trial. J Rheumatol 2011;38:1335-42.

4. Dubey SG, Leeder J, Gaffney K. Physical therapy in anti TNF treated patients with ankylosing spondylitis. Rheumatology 2008;47:1100-1.

J Rheumatol 2011;38:10; doi:10.3899/jrheum.110837 